pag

Business School

WORKING PAPER SERIES

\begin{tabular}{c|l} 
Working Paper & $\begin{array}{l}\text { Motivations et enjeux de l'essaimage } \\
\text { dans les grands groupes français }\end{array}$ \\
$2014-205$ & $\begin{array}{l}\text { Sophie Vallet } \\
\text { Salma Fattoum } \\
\text { Khaled Guesmi }\end{array}$
\end{tabular}

http://www.ipag.fr/fr/accueil/la-recherche/publications-WP.html

IPAG Business School

184, Boulevard Saint-Germain

75006 Paris

France

IPAG working papers are circulated for discussion and comments only. They have not been peer-reviewed and may not be reproduced without permission of the authors. 


\title{
Motivations et enjeux de l'essaimage dans les grands groupes français
}

\author{
Sophie Vallet \\ Consultante en innovation et développement économique, Inno TSD \\ sophie.chevillard@centraliens-lille.org \\ Salma Fattoum \\ Professeur-Chercheur \\ INSEEC Business School, 22 rue Royale 69001 - Lyon \\ sfattoum@groupeinseec.com \\ Khaled Guesmi \\ Professeur-Chercheur \\ Department of Finance, IPAG Research Lab, IPAG Business School \\ 184 Boulevard St Germain ,75006 Paris, France \\ EconomiX - University of Paris Ouest Nanterre La Defense \\ khaled.guesmi@ipag.fr
}

\section{Résumé}

Ayant pris conscience qu'il était stratégique pour elles de s'adapter et d'évoluer, les grandes entreprises françaises ont mis en place de nombreux dispositifs visant à diffuser une dynamique entrepreneuriale. Parmi ces dispositifs, celui de l'essaimage connaît un développement particulier et semble aujourd'hui en pleine mutation aussi bien au niveau des formes qu'il prend que des enjeux qu'il représente.

Le but de ce papier est de comprendre et d'analyser les évolutions du phénomène d'essaimage en se basant sur sa pratique dans dix grands groupes français. A travers ce travail, nous rendons compte de nos investigations et proposons une première modélisation de cette pratique. L'essaimage est pour nous un phénomène multiforme qui concerne trois types de politiques des entreprises : la politique de développement durable et de responsabilité sociale, la politique salariale et la politique d'innovation.

\section{Mots clés}

Essaimage - responsabilité sociale - développement économique local - création d'entreprise. 


\title{
Motives and Dilemmas of Spin-offs in French Large Companies
}

\begin{abstract}
The aim of this paper is to understand and analyze the evolutions of the spin-off process in French large companies based on its practice in ten of them. Through this work, we propose a first modelling of this practice. Spin-off is for us a multiform phenomenon which relates to three types of firm policies: sustainable development and corporate social responsibility, wage policy and innovation policy.
\end{abstract}

\section{Keywords}

Spin-offs - corporate social responsibility - local economic development - entrepreneurship.

Les entreprises évoluent aujourd'hui dans un monde de plus en plus fluctuant. L'ouverture des frontières les confronte à une concurrence toujours plus rude et, pour survivre, elles doivent s'adapter et repenser leurs modèles économiques en prenant en compte les nouvelles contraintes de leur environnement.

En France, les grandes entreprises doivent à la fois répondre aux exigences du marché qui leur imposent une flexibilité et une réactivité toujours plus importantes, mais elles sont également touchées par les questions actuelles de notre société, telles que l'emploi, le développement durable ou encore l'environnement. Elles doivent y apporter des réponses, à la fois pour satisfaire les attentes de leurs clients, mais aussi sous la pression des pouvoirs politiques qui les incitent fortement à agir de façon plus responsable. Ces différentes contraintes sont parfois antinomiques, et les entreprises doivent néanmoins trouver les moyens d'y faire face.

Depuis le début des années 1980, les grandes entreprises françaises montrent un intérêt croissant pour l'entrepreneuriat. En effet, elles ont pris conscience qu'il était important de s'adapter et d'anticiper les évolutions futures. Elles doivent alors développer en leur sein des comportements et attitudes que l'on associe volontiers aux entrepreneurs et qui caractérisent généralement les petites entreprises naissantes : innovation, réactivité, créativité et prise de risque, etc. Nous assistons alors à la mise en place de nombreux dispositifs visant à diffuser une dynamique entrepreneuriale : structures dédiées à l'essaimage, dispositifs de valorisation de la recherche et de l'innovation, fonds d'investissement, etc. Les objectifs de ces pratiques sont multiples : encourager les salariés à développer des projets (en interne mais aussi en externe), développer un réseau de PME innovantes sur lequel l'entreprise pourra s'appuyer et valoriser ses brevets dormants en bénéficiant de la 
flexibilité de petites structures.

Parmi les dispositifs mis en place, celui de cellule essaimage connaît un développement particulier. Né en France dans les années 1980 dans un contexte de récession économique et de restructurations, l'essaimage était à l'origine un moyen de diminuer les effectifs en facilitant le départ des salariés qui avaient un projet de création d'entreprise. Quelques vingt ans plus tard, l'essaimage suscite un regain d'intérêt dans les grands groupes, comme en témoigne la création de Dièse ${ }^{1}$ en 2000 , une association qui réunit une dizaine de grandes entreprises françaises pratiquant l'essaimage et qui œuvre pour sa promotion. L'essaimage semble aujourd'hui en pleine mutation aussi bien au niveau des formes qu'il prend que des enjeux qu'il représente (G. Husson, 2004).

L'essaimage est pratiqué aussi bien dans les PME (E-M. Laviolette, 2005 ;2009) que dans les grandes entreprises (H. Daval, 1998, 2001 ; P. Brenet, 2000). L'intérêt porté aux grands groupes dans notre étude est justifié par le nombre de salariés appartenant à de ce type d'entreprises qui optent pour l'essaimage : environ 1000 par an (APCE, 2012). Nous avons donc rencontré les responsables des cellules essaimage des différentes entreprises membres de Dièse ayant participées à notre recherche. A travers ce travail, nous souhaitons rendre compte de nos investigations. Nous proposons également, dans une démarche exploratoire, une première conceptualisation de cette pratique où nous analysons l'essaimage en termes d'enjeux pour l'entreprise.

Dans une première partie, nous relaterons les principaux travaux qui se sont intéressés au concept d'essaimage dans le contexte français. La deuxième partie sera consacrée à l'exposé de la méthodologie de recherche que nous avons adoptée. Dans une troisième partie, nous présenterons les différents résultats que nous avons mis à jour lors de notre exploration. Enfin, dans la quatrième partie, nous proposerons une première conceptualisation de ce phénomène.

\section{Positionnement contextuel de la recherche : le concept d'essaimage en France}

Depuis les années 1970, l'intérêt pour la création d'entreprises est croissant. En effet, la confiance dans les grands groupes pour dynamiser l'économie s'affaiblit et les nouvelles entreprises apportent l'espoir de la création d'emplois et du renouveau du tissu économique. La création d'entreprises est devenue avec le temps une demande sociale et économique qui implique et intéresse différents acteurs (C. Bruyat, 1993).

Ainsi, les aides à la création d'entreprises se multiplient à tous les stades de développement de la nouvelle organisation et sur toutes ses dimensions : besoins financiers, besoins en conseils et

\footnotetext{
${ }^{1}$ Dièse : Développement de l'initiative et de l'entrepreneuriat chez les salariés. Les membres de l'association sont : Air France, EDF, Sanofi-Aventis, Lafarge, Renault, La Poste, France Télécom, Total, EADS, Schneider, Véolia Environnement
} 
formation, besoins logistiques. Bénéficiant du soutien des pouvoirs publics, les réseaux d'accompagnement se professionnalisent et se structurent.

La création d'entreprise trouve également sa place dans les grandes écoles de commerce et d'ingénieurs qui lancent des programmes pédagogiques pour sensibiliser et préparer leurs étudiants à l'entrepreneuriat (A. Fayolle, 2004). L'élan entrepreneurial gagne aussi les grandes entreprises qui cherchent à développer l'esprit d'initiative de leurs salariés et insuffler une nouvelle dynamique à leur organisation (O. Basso et T. Legrain, 2004). Aussi, de nombreux dispositifs voient le jour pour favoriser l'éclosion de nouveaux projets et la création de nouvelles entreprises. L'essaimage est l'un de ces dispositifs. Il consiste pour une entreprise à aider un ou plusieurs de ses employés à créer leur propre entreprise.

Traduction incomplète du terme «spin-off» (F. Pirnay, 1998, p.3), le concept d'essaimage a pris, en France, une signification particulière. Il a fait l'objet de différentes études (C. Bruyat, 1987 ; L. Danvert, 1988 ; B. Sire, 1988 ; J. Bertherat, 1989 ; M. Lafrance, 1994 ; A. Belley, L. Dussault et J. Lorrain, 1997 ; H. Daval, 1998, 2001 ; F. Pirnay, 1998, 2001 ; P. Brenet, 2000 ; E-M. Laviolette, 2003, 2005, 2009) qui ont tenté de mieux expliciter ce phénomène et de l'enrichir. Il faut noter que le concept d'essaimage renvoie à des pratiques différentes en France et aux Etats-Unis. De ce fait, la littérature anglo-saxonne s'intéressant à ce concept n'est pas toujours bien adaptée au contexte français, car elle renvoie à un phénomène différent. La littérature sur l'essaimage dans le contexte français n'est toutefois pas très importante.

Les principaux travaux qui s'y réfèrent adoptent souvent le point de vue du salarié qui part créer une entreprise avec l'aide de son employeur. Ainsi, P. Brenet (2000) définit l'essaimage comme "la création d'une entreprise par une personne (ou une équipe) qui a quitté son entreprise d'origine, avec l'existence d'un lien formel ou d'influence entre ces deux entreprises ». De même, H. Daval (1998) met l'accent sur le rôle de l'entreprise dans l'accompagnement de son salariéporteur de projet.

Plusieurs chercheurs ont proposé une typologie des différentes pratiques d'essaimage mises en place par les entreprises. Ainsi, l'essaimage « à la française »- du fait du contexte dans lequel il a émergé - est souvent réduit à sa dimension sociale, c'est-à-dire à la gestion des sureffectifs (P. Brenet, 2000). Pourtant, les finalités poursuivies par une organisation, dès lors qu'elle s'engage dans une politique d'essaimage, sont multiples. Historiquement, une première typologie s'est basée sur les finalités poursuivies par chaque organisation et a mis en avant le fait qu'elles diffèrent, selon que son contexte économique lui est favorable ou pas. De ce constat, B. Sire (1988), C. Bruyat (1988) ainsi que A. Belley et al. (1997) font la distinction entre l'essaimage à froid (contexte favorable) et l'essaimage à chaud (contexte défavorable). 
L'essaimage à froid est une politique que mène délibérément une organisation afin de stimuler l'esprit entrepreneurial chez des membres de son personnel dans le but de susciter parmi ces derniers des projets, en particulier d'innovation. Cette politique d'essaimage doit son appellation à son caractère volontariste et est généralement associée aux organisations en bonne santé en raison des moyens que ce type de politique nécessite (B. Sire, 1988; C. Bruyat, 1988).

L'essaimage à chaud est une politique à laquelle fait appel une organisation se trouvant en difficulté, dans le but de réduire l'effectif de son personnel.

D'autres finalités sont associées à l'essaimage, principalement dans le cas d'une politique «à froid ». Ainsi, l'essaimage peut être perçu comme un moyen de développer une relation, un partenariat basé sur la mise en commun des compétences et des ressources nécessaires à la croissance (H. Daval, 1998). Les objectifs inhérents à cette pratique peuvent également concerner le fait de favoriser le développement d'une culture entrepreneuriale, aussi bien en interne pour gérer les effectifs et redynamiser les équipes (H. Daval, 1998), qu'en externe pour développer des relations basées sur les synergies inter-entreprises (W-J. Dennis et B-D. Phillips, 1990), éléments préalables à la formation de réseaux (S. Birley, 1985). Une organisation peut enfin avoir recours à une politique d'essaimage pour valoriser son image (H. Daval, 1998).

Les principaux travaux qui se réfèrent à l'essaimage mettent le focus sur le résultat du processus -la création ou la reprise d'une entreprise- et sur les synergies que cette nouvelle entité est susceptible de développer avec son entreprise d'origine. Nous avons choisi de positionner notre recherche sur 1'«entreprise-mère » elle-même et de nous intéresser aux enjeux que représente pour elle l'essaimage. De ce fait, notre intérêt ne s'est pas porté uniquement sur le résultat de l'essaimage, à savoir l'entreprise créée ou reprise ; nous avons adopté une démarche plus globale pour comprendre les finalités attendues par les différents acteurs qui pratiquent l'essaimage.

\section{Méthodologie}

Notre étude a été effectuée dans le cadre d'un projet auquel nous étions associées et qui consistait à décrire les pratiques d'essaimage dans les grands groupes français. Notre étude exploratoire concerne dix entreprises françaises réparties dans différents secteurs d'activité ${ }^{2}$. Les données ont été collectées par des entretiens semi directifs auprès des personnes responsables de l'essaimage dans les entreprises. Nous avons effectué au total 10 entretiens. Ils ont été réalisés à l'aide d'un guide d'entretien comprenant quatre thèmes principaux: l'entrepreneuriat au sens large au sein de

\footnotetext{
${ }^{2}$ Les dix entreprises sont : le CEA et neuf entreprises membres de Dièse, à savoir, EDF, France Télécom, Air France, SanofiAventis, La Poste, Lafarge, Total, Renault, EADS, Schneider, Véolia Environnement. Le passage par une association était indispensable pour pouvoir approcher ces entreprises, pratique d'ailleurs utilisée dans d'autres études reconnues sur l'essaimage (Daval, 2001 et Laviolette, 2005).
} 
l'entreprise, c'est-à-dire les attitudes générales face à l'innovation et à l'émergence de nouveaux projets ; la vision générale de la structure d'essaimage et de la place qu'elle occupe au sein de l'entreprise ; l'organisation interne de cette structure ; et enfin, le dispositif d'accompagnement. Les données ont été recueillies en face-à-face. La durée moyenne de chaque entretien a été de deux heures et tous ont été enregistrés sous format numérique. Les informations collectées ont été complétées par des données secondaires, notamment, des rapports et des documents de suivi de l'activité essaimage qui nous ont été fournis par certaines des entreprises rencontrées.

La méthode d'analyse qui nous a semblé la plus adéquate pour le traitement des données collectées est celle de l'analyse de contenu. Cette méthode nous a permis de nous intéresser au sens des données et aux interprétations des personnes interviewées.

La pratique d'essaimage n'a cessé d'évoluer depuis son apparition et est essentiellement pratiquée par des grands groupes industriels. Il nous a donc semblé pertinent de choisir pour notre échantillon des entreprises de cette population afin d'essayer de répondre à notre problématique qui est de comprendre les enjeux de l'essaimage. Neuf éléments de notre échantillon sont membres de Dièse. Toutes ces entreprises ont une démarche proactive puisqu'elles ont fait le choix de devenir membre d'une association (Dièse) qui cherche à promouvoir les pratiques d'essaimage. Par ailleurs, le CEA (Centre d'Énergie Atomique) est un organisme financé à hauteur de 50\% par des fonds publics qui pratique de façon continue l'essaimage pour valoriser ses brevets. Il nous a donc semblé intéressant de l'intégrer à notre échantillon pour l'enrichir.

\section{Résultats}

Dans cette partie, nous allons présenter et analyser les résultats obtenus à la suite des entretiens que nous avons effectués. D'abord, l'historique de chacune des entreprises rencontrées nous permettra de mettre en exergue les faits saillants de la naissance des cellules essaimage des entreprises de notre échantillon. Nous nous intéresserons ensuite aux différents facteurs de motivations de l'essaimage ainsi qu'aux dispositifs connexes.

\section{Historique des entreprises rencontrées}

Le tableau 1 résume l'organisation des cellules essaimage dans les différentes entreprises ainsi que l'effectif qui en a la charge. 
Tableau 1 - Récapitulatif de l'organisation de l'essaimage*

\begin{tabular}{|c|c|c|c|}
\hline Entreprise & $\begin{array}{l}\text { Date de } \\
\text { création }\end{array}$ & Positionnement hiérarchique & Effectif \\
\hline $\begin{array}{l}\text { Sanofi- } \\
\text { Aventis }\end{array}$ & 1986 & direction des ressources humaines & 3 personnes au sein de la cellule. \\
\hline EDF & $\begin{array}{l}\text { création en } \\
\quad 1990, \\
\text { réorganisation } \\
\text { en } 2000\end{array}$ & direction de l'emploi & $\begin{array}{l}\text { la cellule s'appuie sur une dizaine de conseillers } \\
\text { répartis au sein de l'entreprise. }\end{array}$ \\
\hline $\begin{array}{l}\text { France } \\
\text { Télécom }\end{array}$ & 1998 & $\begin{array}{l}\text { RH groupe mais création d'une entité } \\
\text { appelée Recrutement Mobilité Carrière } \\
\text { à laquelle la cellule va être rattachée }\end{array}$ & 7 délégués régionaux +3 recrutements en cours. \\
\hline La Poste & 1986 & direction des ressources humaines & $\begin{array}{l}2 \text { personnes et un secrétariat au sein de la } \\
\text { cellule, et un réseau de "conseillers mobilité" à } \\
\text { travers l'entreprise. }\end{array}$ \\
\hline $\begin{array}{c}\text { Air } \\
\text { France }\end{array}$ & 1995 & direction des ressources humaines & $\begin{array}{l}\text { l'essaimage est pris en charge par environ } 10 \\
\text { personnes, à temps plein ou temps partiel. }\end{array}$ \\
\hline Renault & 1984 & Secrétaire Général & 1 personne. \\
\hline EADS & 1996 & $\begin{array}{c}\text { au sein de la filiale EADS } \\
\text { développement } \\
\text { rattachée à la direction des ressources } \\
\text { humaines }\end{array}$ & $\begin{array}{l}-1 \text { personne à temps plein dans les grandes } \\
\text { filiales. } \\
-1 \text { personne à temps partiel dans les petites } \\
\text { filiales. }\end{array}$ \\
\hline Total & 1986 & direction du développement régional & 2 personnes. \\
\hline Lafarge & 1986 & $\begin{array}{l}\text { RH France. Activité transversale au } \\
\text { groupe }\end{array}$ & $\begin{array}{l}1 \text { personne en charge de l'essaimage et des } \\
\text { relais auprès des Responsables RH dans les } \\
\text { différentes activités du groupe. }\end{array}$ \\
\hline CEA & 1986 & $\begin{array}{l}\text { direction de la valorisation qui dépend } \\
\text { de la direction de la recherche } \\
\text { technologique }\end{array}$ & $\begin{array}{l}3 \text { personnes (à temps plein ou temps partiel) en } \\
\text { charge de l'essaimage qui s'appuient sur des } \\
\text { correspondants dans les différents centres du } \\
\text { CEA. }\end{array}$ \\
\hline
\end{tabular}

\section{Les facteurs de motivation de l'essaimage}

Lors de nos entretiens, nous nous sommes intéressés aux raisons pour lesquelles ces grands groupes industriels pratiquaient l'essaimage. Le tableau 2 résume les différents facteurs de motivation cités par les entreprises rencontrées.

Il est intéressant de constater que l'essaimage reflète le positionnement et les priorités de l'entreprise en termes de politique économique et sociale. Il est l'une des vitrines de l'entreprise. Sanofi- Aventis et le CEA sont deux exemples où ce positionnement est très clair :

\footnotetext{
* Ce tableau a été réalisé à partir des informations recueillies en entretien, les données ne proviennent donc pas d'une communication institutionnelle des entreprises.
} 
Tableau 2 - Facteurs de motivation à la création de l'essaimage

\begin{tabular}{|c|c|}
\hline Entreprise & Facteurs de motivation pour mettre en place la cellule \\
\hline Sanofi-Aventis & $\begin{array}{l}\text { - favoriser l'initiative des salariés. } \\
\text { - attitude citoyenne pour soutenir la création d'emplois. } \\
\text { - gérer les ressources humaines. } \\
\text { - argument de recrutement. }\end{array}$ \\
\hline EDF & $\begin{array}{l}\text { - contribuer au développement économique (la responsabilité sociétale). } \\
\text { - dimension stratégique (qui est mieux placé que quelqu'un qui a développé une activité en } \\
\text { interne qui intéresse le groupe pour la porter à l'externe). } \\
\text { - respiration de l'entreprise (l'entreprise a intérêt à accompagner ses salariés y compris sur } \\
\text { des projets à l'externe). }\end{array}$ \\
\hline $\begin{array}{l}\text { France } \\
\text { Télécom }\end{array}$ & $\begin{array}{l}\text { - développer un esprit entrepreneurial parmi les salariés. } \\
\text { - élan de la R\&D pour valoriser la recherche et les brevets non exploités en interne. } \\
\text { - élan de la part des RH pour développer une politique de développement et de maintien de } \\
\text { l'économie locale. } \\
\text { - augmenter le turn-over. }\end{array}$ \\
\hline La Poste & - encourager les porteurs d'une idée indépendante à aller au bout de l'idée. \\
\hline AIR France & - essaimage à chaud et gestion du sureffectif. \\
\hline Renault & - gestion des ressources humaines. \\
\hline EADS & $\begin{array}{l}\text { - initiative de Aérospatiale pour agir en entreprise citoyenne en s'impliquant sur les bassins } \\
\text { d'emplois où elle est implantée. }\end{array}$ \\
\hline Total & $\begin{array}{l}\text { - gérer les ressources humaines. } \\
\text { - augmenter le turn-over. } \\
\text { - contribuer au rayonnement de Total (en terme d'image). }\end{array}$ \\
\hline Lafarge & $\begin{array}{l}\text { - gestion des ressources humaines (notamment lors de la reconfiguration de sites). } \\
\text { - développer l'esprit d'initiative. }\end{array}$ \\
\hline CEA & $\begin{array}{l}\text { - une des activités de la stratégie de la valorisation du CEA (essaimage technologique). } \\
\text { - favoriser un esprit entrepreneurial (pour valoriser la recherche). } \\
\text { - gestion des ressources humaines (essaimage de salariés). }\end{array}$ \\
\hline
\end{tabular}

- Chez Sanofi, l'essaimage est né en 1986 de la volonté du président du groupe d'insuffler un esprit d'initiative au sein de son entreprise. Il partait du principe qu'il fallait aider les gens qui avaient un projet à le réaliser, que ce dernier soit en interne ou en externe. Pour faire en sorte que l'essaimage véhicule une image positive aux salariés, une structure annexe, la filiale Sopran, a été créée pour traiter les cas de sauvegarde de l'emploi. De ce fait une distinction très nette est faite entre essaimage à chaud et essaimage à froid. Aujourd'hui, aux dires de son responsable, "l'essaimage est une politique qui incarne les valeurs de la maison : le courage, l'audace, le respect, la solidarité, la performance, l'innovation ».

- Au sein du CEA, l'essaimage est rattaché à la direction de la valorisation et est considéré comme un outil de valorisation de la recherche industrielle. L'accompagnement a été structuré autour d'une hiérarchie des projets en quatre classes, suivant que le projet comporte une forte dimension technologique (avec une technologie issue du CEA) ou non.

La position de l'essaimage au sein de Total et EADS reflète également les priorités de l'entreprise. 
En effet, dans ces deux entreprises, l'essaimage est rattaché aux filiales en charge du développement économique local. Il fait donc partie intégrante de cette politique.

Lorsque l'essaimage est motivé par des arguments de gestion des ressources humaines (par exemple au sein de la Poste ou d'EDF), le positionnement de la cellule essaimage est également cohérent avec les objectifs qui lui sont fixés, puisqu'elle est rattachée à une structure RH (direction des ressources humaines, direction de l'emploi) qui sert de relais à sa diffusion en interne.

\section{Les projets développés et l'accompagnement des salariés}

Nous nous sommes intéressés aux projets accompagnés par les cellules essaimage, afin d'établir un comparatif du nombre de projets qui aboutissent à une création ou une reprise d'entreprise dans les différentes entreprises. Nous avons pu noter que le nombre d'entreprises créées variait d'une dizaine à une soixantaine par an. Le nombre de départ de salariés pour création d'entreprise représente environ 1\%o de l'effectif total dans différents groupes que nous avons rencontrés. Ces chiffres sont donc peu élevés par rapport à la masse salariale de ces entreprises et montrent que l'essaimage pratiqué est bien offensif.

Concernant l'accompagnement réalisé au sein des cellules essaimage, celui-ci est assez similaire dans toutes les entreprises. Ainsi, quels que soient les facteurs de motivation de l'essaimage, le processus d'accompagnement est le même. Cela s'explique par le fait que les projets menés sont assez similaires quelle que soit l'entreprise. Il s'agit la plupart du temps de la création ou de la reprise d'une petite entreprise de service de proximité. L'accompagnement consiste donc en un suivi du porteur de projet pendant la phase de développement du projet, une aide à la rédaction de son business plan ainsi qu'un soutien financier. Les porteurs de projets sont également mis en relation avec différents acteurs, dont les réseaux d'accompagnement comme les Chambres de Commerce et d'Industrie (CCI) ou les boutiques de gestion, mais aussi d'anciens créateurs ou des organismes financiers. Le CEA, qui accompagne davantage de projets innovants, a mis en place une procédure d'accompagnement plus complexe. Il s'appuie notamment sur les incubateurs publics et propose des aides financières plus élevées, pour permettre des levées de fonds plus importantes.

\section{Des dispositifs connexes}

L'objectif de notre exploration était de comprendre comment l'essaimage s'insérait au sein des entreprises. Aussi, nous nous sommes intéressés aux autres dispositifs qui, dans l'entreprise, favorisaient l'entrepreneuriat et s'appuyaient sur les mêmes facteurs de motivations que l'essaimage. Le tableau 3 résume l'ensemble de ces dispositifs.

Nous pouvons noter que pour la plupart des entreprises de notre échantillon, l'essaimage n'est pas un dispositif isolé au sein de l'organisation. Il est lié à d'autres dispositifs qui partagent des valeurs similaires. 
Ainsi, certaines entreprises ont mis en place d'autres dispositifs de développement durable et de responsabilité sociale : c'est le cas de Total qui a un programme important d'aide au développement des PME. De même, Sanofi, Lafarge et EADS cherchent à jouer un rôle pour aider au développement local. Ils financent et accompagnent des projets de création ou de développement d'entreprises conduisant à créer des emplois.

Tableau 3 - Les autres dispositifs favorisant l'entrepreneuriat au sein des entreprises

\begin{tabular}{|c|c|c|}
\hline & $\begin{array}{l}\text { Autres dispositifs dans l'entreprise qui } \\
\text { partagent les mêmes valeurs }\end{array}$ & Actions menées \\
\hline $\begin{array}{l}\text { Sanofi- } \\
\text { Aventis }\end{array}$ & Filiale Sopran & $\begin{array}{l}\text { - actions qui s'inscrivent dans le développement durable. } \\
\text { - actions de capital risque pour aider à la reconversion } \\
\text { industrielle et au ré-emploi des sites que l'entreprise quitte. } \\
\text { - actions menées auprès des PME et créateurs d'entreprises } \\
\text { locaux. }\end{array}$ \\
\hline EDF & \multicolumn{2}{|l|}{ cellule dédiée à l'essaimage stratégique } \\
\hline \multirow{3}{*}{$\begin{array}{l}\text { France } \\
\text { Télécom }\end{array}$} & dispositif de formation & $\begin{array}{l}\text { formation des cadres pour qu'ils développent un esprit } \\
\text { d'initiative. }\end{array}$ \\
\hline & dispositif pour les jeunes embauchés & $\begin{array}{l}\text { formation des jeunes qui sortent de grandes écoles pour leur } \\
\text { faire développer un élan entrepreneurial. }\end{array}$ \\
\hline & fond d'investissement "innovacom'" & $\begin{array}{l}\text { prise de participation dans des entreprises technologiques en } \\
\text { communication. }\end{array}$ \\
\hline La Poste & \multicolumn{2}{|l|}{ Aucun autre dispositif } \\
\hline AIR France & SODESI : filiale de reconversion & $\begin{array}{l}\text { - aide au développement économique local. } \\
\text { - bilan de compétences. } \\
\text { - prestations de service en matière de ressources humaines pour } \\
\text { Air France. }\end{array}$ \\
\hline Renault & \multicolumn{2}{|l|}{ Aucun autre dispositif } \\
\hline EADS & $\begin{array}{l}\text { EADS développement } \\
\text { (filiale à laquelle appartient la cellule } \\
\text { essaimage) }\end{array}$ & $\begin{array}{l}\text { aider au développement des PME locales pour favoriser } \\
\text { l'embauche. }\end{array}$ \\
\hline Total & direction du développement régional & $\begin{array}{l}\text { accompagnement de PME sur les territoires sur lesquels } \\
\text { l'entreprise est implantée : } \\
\text { - aides techniques ; } \\
\text { - aides financières (prêts) ; } \\
\text { - aide au développement à l'international. }\end{array}$ \\
\hline \multirow[t]{2}{*}{ Lafarge } & $\begin{array}{l}\text { l'animation RH France s'occupe aussi de } \\
\text { développement économique local (dans } \\
\text { toute l'Europe) }\end{array}$ & $\begin{array}{l}\text { aides financières aux entreprises en développement qui vont } \\
\text { créer de l'emploi. }\end{array}$ \\
\hline & \multicolumn{2}{|l|}{ l'essaimage est adossé à la cellule mobilité } \\
\hline \multirow{5}{*}{ CEA } & dispositif de valorisation de la technologie & partenariats ou prestations avec des industriels. \\
\hline & $\begin{array}{l}\text { accompagnement de porteurs de projets non } \\
\text { salarié du CEA }\end{array}$ & $\begin{array}{l}\text { - accompagnement des porteurs de projet non CEA mais dont le } \\
\text { projet s'appuie sur une technologie du CEA : } \\
\text { - orientation vers un incubateur ; } \\
\text { - contrat de R\&D ou d'exploitation d'un brevet ; } \\
\text { - négociation possible d'un hébergement au sein d'un } \\
\text { laboratoire pendant la période d'incubation. }\end{array}$ \\
\hline & fond d'investissement CEA valorisation & $\begin{array}{l}\text { prise de participation dans les projets qui s'appuient sur une } \\
\text { technologie issue du CEA. }\end{array}$ \\
\hline & \multicolumn{2}{|l|}{ concours d'idées } \\
\hline & \multicolumn{2}{|c|}{ programme de sensibilisation à l'entrepreneuriat (avec EM Lyon) } \\
\hline
\end{tabular}

Favoriser un esprit entrepreneurial auprès des salariés n'est pas non plus réalisé par la seule cellule essaimage. Chez France Télécom, ou encore au CEA, différents dispositifs de formation visent à 
sensibiliser les salariés à l'entrepreneuriat pour les faire évoluer vers un comportement plus entrepreneurial. Enfin, la gestion de l'innovation est également la finalité d'autres dispositifs que la cellule essaimage.

Ainsi, les entreprises dont le potentiel d'innovation est un moteur de développement, comme EDF ou France Télécom, ont créé leur propre fond d'investissement, afin de s'entourer de nouvelles entreprises technologiques développant une innovation en lien avec leur cœur de métier.

Le CEA dispose lui aussi d'un fond d'investissement, mais dans une optique de valorisation, puisqu'il prend des participations dans les entreprises qui s'appuient sur une technologie issue du CEA. Il existe donc, au sein des organisations, différents dispositifs connexes qui contribuent à répondre aux problématiques actuelles des entreprises, tels que le développement durable, l'entrepreneuriat ou l'innovation. La cellule essaimage n'est qu'un dispositif parmi ceux-ci.

\section{Conceptualisation : une catégorisation de l'essaimage}

Notre exploration nous a permis de déterminer, en partie, quelles étaient les attentes des entreprises vis-à-vis de l'essaimage. Les facteurs de motivation mentionnés par les personnes rencontrées se sont révélés variables d'une entreprise à l'autre (cf. tableau 3).

Nous pensons que ces facteurs de motivation peuvent s'articuler autour de trois dimensions :

- la gestion des ressources humaines ;

- la responsabilité sociale et le développement économique local ;

- la valorisation technologique et la gestion de l'innovation.

En effet, chaque entreprise semble s'appuyer sur l'une (ou plusieurs) de ces dimensions pour expliquer ce qui a conduit leur entreprise à mettre en place l'essaimage et à continuer à le pratiquer. Le tableau 4 reprend les différents facteurs de motivation cités lors de nos entretiens, que nous avons classés selon ces trois catégories.

En outre, comme notre exploration nous a permis de le mettre à jour, la cellule essaimage ne constitue souvent qu'un élément d'une structure plus large au sein de l'entreprise à laquelle elle est adossée. Ainsi, l'essaimage ne se limite pas à une pratique entrepreneuriale dont le but est uniquement la création d'entreprises par des salariés, mais elle est généralement le reflet d'une politique plus générale de l'entreprise. Les dispositifs connexes qui existent dans la plupart des entreprises peuvent être catégorisés de la même façon que les objectifs des cellules essaimage et témoignent des politiques générales des entreprises. Certes, le nombre de projets accompagnés chaque année et les moyens mobilisés pour l'essaimage dans les grandes entreprises ne sont pas très importants, mais le fait que de telles pratiques existent et l'effort de mutualisation dont elles font l'objet, montrent une réflexion des entreprises sur d'autres questions que leur seule rentabilité 
économique, et font ainsi de l'essaimage une pratique au cœur des enjeux de l'entreprise. La conceptualisation que nous proposons est une représentation de ce constat (figure 1).

Tableau 4 - Facteurs de motivation pour pratiquer l'essaimage

\begin{tabular}{|c|c|}
\hline $\begin{array}{l}\text { Gestion des ressources } \\
\text { humaines }\end{array}$ & $\begin{array}{ll}- & \text { Favoriser l'initiative des salariés. } \\
\text { - } & \text { Développer un esprit entrepreneurial parmi les salariés. } \\
\text { - } & \text { Favoriser les projets des salariés qui ont développé une activité en interne } \\
\text { - } & \text { pour la porter à l'externe. } \\
\text { - } & \text { Augmenter le turn-over. } \\
\text { - } & \text { Argument de recrutement. } \\
\text { - } & \text { Encourager les porteurs d'une idée indépendante à aller au bout de l'idée. } \\
\text { - } & \text { Respiration de l'entreprise (l'entreprise a intérêt à accompagner ses salariés } \\
& \text { y compris sur des projets à l'externe). } \\
\text { - } & \text { Essaimage à chaud et gestion du sureffectif. }\end{array}$ \\
\hline $\begin{array}{l}\text { Développement durable } \\
\text { et responsabilité sociale }\end{array}$ & 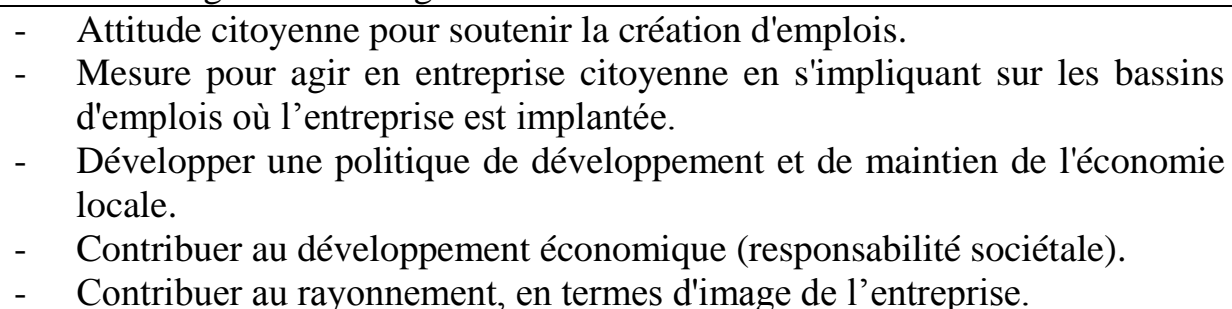 \\
\hline Gestion de l'innovation & $\begin{array}{ll}- & \text { Valoriser la recherche et les brevets non exploités en interne. } \\
\text { - } & \text { Stratégie de la valorisation (essaimage technologique). }\end{array}$ \\
\hline
\end{tabular}

Nous pouvons mettre à jour des caractéristiques différentes pour les trois catégories de cellules essaimage - et des dispositifs connexes auxquels ils sont adossés : des dispositifs hétérogènes en termes de moyens et de ressources, un positionnement dans l'organigramme différent d'une entreprise à l'autre et corrélé aux objectifs de la cellule essaimage, des aides variables dépendantes de critères définis par les entreprises (appartenance ou non à l'entreprise, technologie issue de l'entreprise, etc.), un lien plus ou moins fort entre la nouvelle entreprise créée et l'entreprise-mère, etc. Mais la différence que nous jugeons principale entre ces trois catégories se situe au niveau du public visé. Nous avons en effet déterminé trois acteurs qui interagissent avec l'entreprise via les dispositifs entrepreneuriaux: le salarié, le territoire (c'est-à-dire le bassin d'emplois auquel appartient l'entreprise) et l'entreprise elle-même. De plus, ces trois acteurs sont associés à un facteur de motivation en particulier :

- la gestion des ressources humaines est à destination du salarié. C'est lui le bénéficiaire de la politique et des dispositifs mis en place, et c'est à travers lui que l'entreprise réalise ses objectifs en terme de prise d'initiative, de respiration de l'entreprise, etc. Ainsi, lorsque le choix de mettre en place une cellule essaimage est guidé par la gestion des ressources humaines, la communication est essentiellement interne à l'entreprise, via l'intranet, les responsables RH et le magazine interne. De même, les résultats de l'essaimage (nombre de créations, best practice et réussite exemplaire, etc.) 
sont aussi communiqués en interne. Ils servent d'exemples et sont le vecteur pour diffuser un esprit entrepreneurial ;

- la responsabilité sociale, quant à elle, a pour cible l'environnement de l'entreprise, c'est-à-dire les territoires et les bassins d'emplois sur lesquels elle est implantée. Il s'agit d'une relation gagnant/gagnant entre l'entreprise et son environnement : l'entreprise favorise le développement économique et la création d'emplois afin de minimiser son impact sur les territoires, notamment lorsqu'elle est amenée à les quitter ;

- enfin, la gestion de l'innovation vise l'entreprise elle-même. C'est elle le bénéficiaire des avancées technologiques. Nous pouvons néanmoins distinguer deux cas de figures, selon que l'entreprise cherche à valoriser ses innovations développées en interne ou qu'elle cherche à développer des innovations en lien avec son cœur de métier. Dans le premier cas, l'innovation est une part importante de la richesse de l'entreprise, et elle doit donc mettre en place une stratégie de valorisation de cette richesse. Il ne s'agit pas uniquement de déposer des brevets, mais également de les exploiter, à la fois en interne, mais aussi en externe, par exemple à travers un fond de capital risque, des partenariats industriels, etc. Dans le deuxième cas, l'entreprise doit innover pour conserver sa position concurrentielle. Elle peut alors à la fois développer ses propres innovations, mais aussi s'appuyer sur des start-ups innovantes en bénéficiant de leur flexibilité.

Nous pouvons alors définir trois formes d'entrepreneuriat qui reflètent les divers objectifs et impliquent des acteurs différents :

- l'entrepreneuriat salarial, défini par des objectifs de gestion des ressources humaines et qui concerne les salariés d'une entreprise.

- l'entrepreneuriat citoyen, qui concerne le territoire où est implanté l'entreprise et qui est défini par une politique de développement durable et de responsabilité sociale de cette dernière.

- l'entrepreneuriat stratégique, qui concerne l'entreprise elle-même et répond à une volonté de favoriser et valoriser l'innovation. 
Figure 1 - La cellule essaimage : au cour des enjeux de l'entreprise

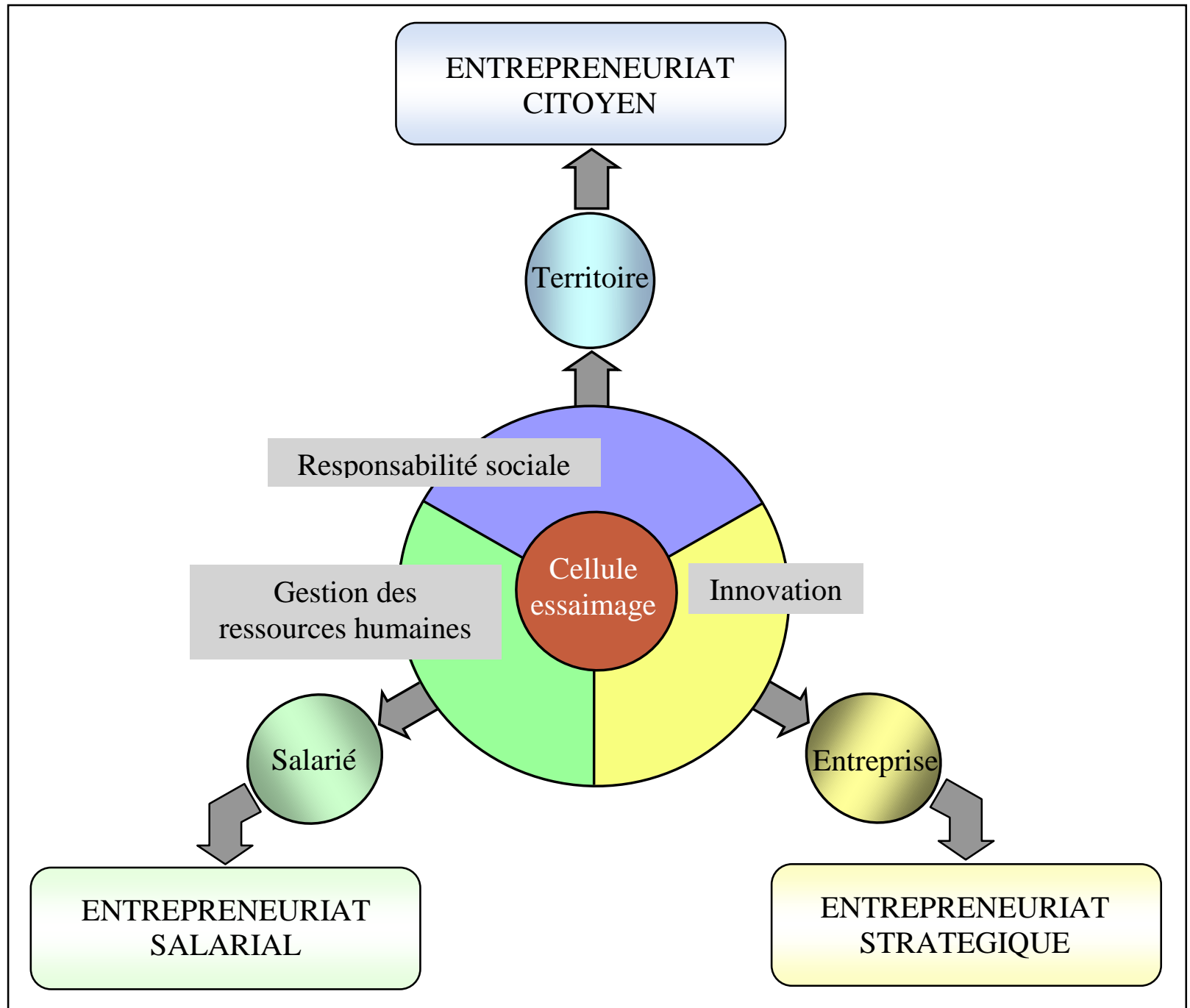

Notre conceptualisation nous permet donc de visualiser la cellule essaimage et les dispositifs connexes comme des éléments d'articulation entre les logiques antagonistes de ces trois acteurs :

- La logique de l'entreprise qui cherche à développer ses activités et asseoir sa position concurrentielle et son rayonnement commercial ;

- La logique du salarié qui aspire à voler de ses propres ailes ;

- La logique du territoire pour qui la création d'entreprise est synonyme de richesse et de développement économique.

C'est au niveau de cette articulation que se situent les enjeux de l'entreprise. En effet, les pressions de l'opinion publique et des pouvoirs politiques qu'elle subit la conduisent à respecter, outre ses propres intérêts, ceux de ses partenaires. Elle se doit donc de les prendre en compte. L'essaimage et l'ensemble des dispositifs entrepreneuriaux existants dans l'entreprise participent alors à allier ces intérêts divergents. De plus, la cohérence du système vis-à-vis des différentes parties prenantes permet d'assurer une dynamique positive, c'est-à-dire un équilibre entre les besoins et objectifs de l'entreprise et les exigences des différents acteurs. Sur la figure 2, nous avons placé les entreprises 
de notre échantillon. Nous pouvons ainsi mettre en évidence plusieurs groupes d'entreprises en fonction des objectifs recherchés : pour le premier groupe (bleu), la cible prioritaire est le salarié, et l'objectif principal de l'essaimage est la gestion des ressources humaines. Le deuxième groupe (rouge) agit selon deux directions, le territoire et le salarié. L'essaimage est alors au cœur de leur politique de développement durable et de leur politique salariale. Le troisième groupe (vert) allie politique salariale et gestion de l'innovation. Enfin, le quatrième groupe (marron) cible l'ensemble des objectifs et des acteurs : l'essaimage est au cœur des trois politiques.

Figure 2 - Mapping des entreprises de notre échantillon

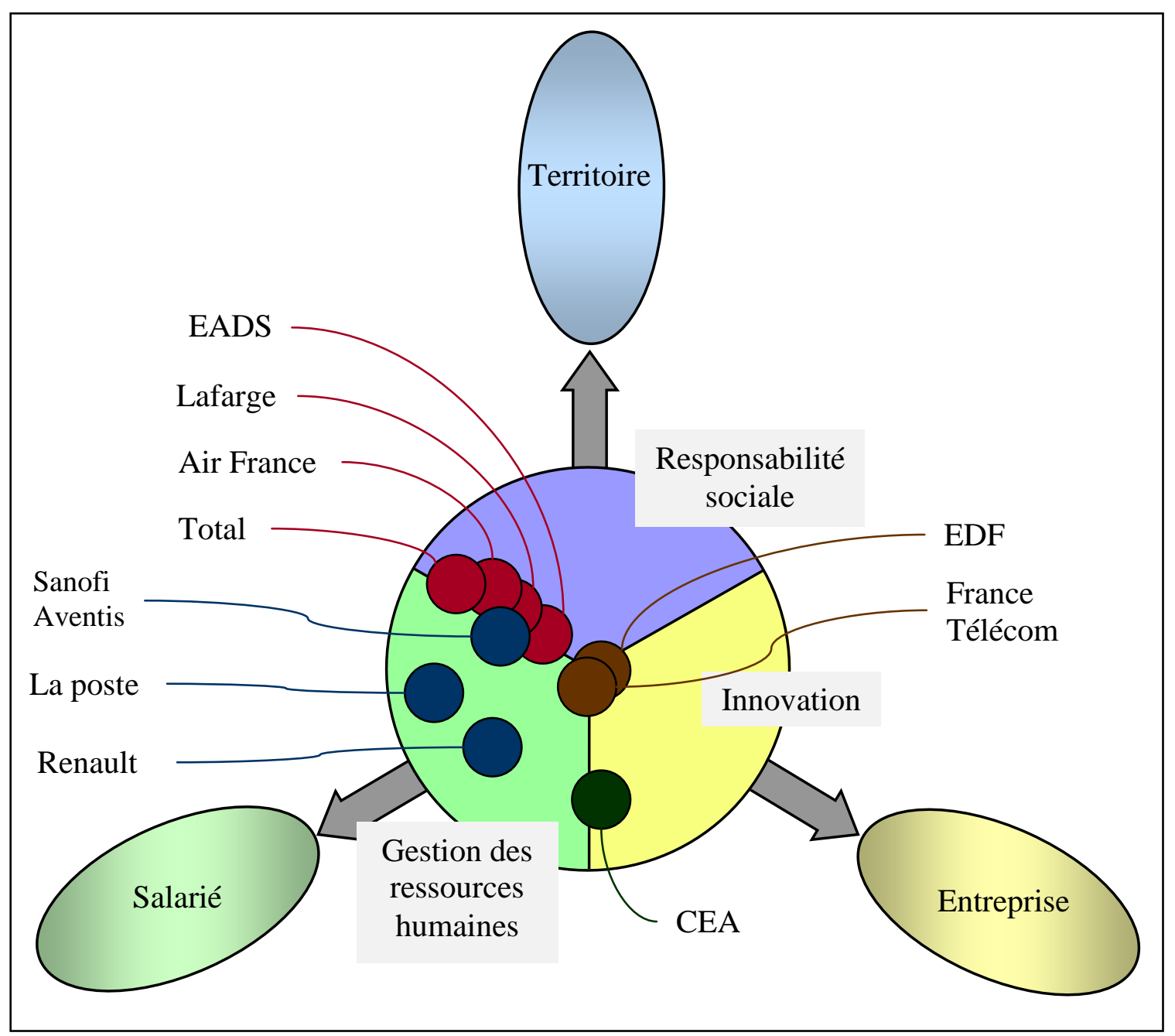

\section{Conclusion}

La conceptualisation que nous avons proposée permet d'apporter un nouvel éclairage sur l'essaimage. Outre le fait qu'il s'agit d'un dispositif entrepreneurial (dans le sens où il favorise la création ou la reprise d'entreprises), l'essaimage est pour nous au cœur des enjeux actuels de 
l'entreprise, car il reflète, au même titre que d'autres dispositifs, les priorités des grands groupes français. Toutes les entreprises n'ont pas les mêmes attentes vis-à-vis de l'essaimage et nous avons dégagé trois politiques auxquelles les cellules essaimage font écho: une politique de développement durable et de responsabilité sociale, une politique d'innovation et une politique salariale.

Cette vision de l'essaimage est pour nous un point de départ pour analyser les dispositifs qui favorisent l'entrepreneuriat au sein de la grande entreprise sous un autre angle que celui seul du résultat (entreprise créée, opportunité saisie, processus conduit). Adopter le point de vue de l'entreprise nous a permis d'envisager cette pratique de manière plus globale, en recherchant les synergies qu'elle peut développer à l'intérieur de l'entreprise, mais aussi avec ses partenaires.

Le travail que nous avons réalisé est bien sûr conditionné à notre échantillon. La principale limite concerne la composition de notre échantillon. Il contient en effet neufs entreprises membres de Dièse. Les observations que nous avons faites peuvent donc être biaisées du fait que les personnes que nous avons rencontrées échangent sur leurs pratiques au sein de l'association. En outre, leur vision de l'essaimage peut également refléter à la fois la position de leur entreprise, mais aussi la position de Dièse.

Une deuxième limite concerne les personnes que nous avons rencontrées. En effet, faute de temps, les responsables des cellules essaimage ont été nos uniques interlocuteurs dans les entreprises. De ce fait, nous avons eu accès à la vision d'une seule personne sur les enjeux de l'essaimage. Il aurait été intéressant de comparer cette vision à celle des personnes essaimées et des autres instances de l'entreprise en lien avec l'essaimage.

Ce travail peut en outre conduire à différents approfondissements. Il s'agit d'un premier travail exploratoire qui mériterait d'être étudié de façon confirmatoire, et notamment auprès d'autres entreprises que celles membres de Dièse.

En outre, la conceptualisation que nous avons proposée est un premier pas pour explorer l'essaimage en tant qu'outil de développement durable, de gestion des ressources humaines et d'innovation. Il serait intéressant de poursuivre les investigations sur chacune des dimensions et de comprendre les synergies entre l'essaimage et les autres dispositifs de la politique en question. 


\section{Bibliographie}

Agence Pour la Création d'Entreprise (2012), Favoriser la création et le reprise d'entreprises par les salariés, Guide opérationnel d'essaimage, $3^{\mathrm{ème}}$ édition, $110 \mathrm{p}$.

Basso O., Legrain T. (2004), La dynamique entrepreneuriale dans les grands groupes, Institut de l'entreprise.

Belley A., Dussault L. et Lorrain J. (1997), L'essaimage : une stratégie délibérée de développement économique, Fondation de l'Entrepreneurship, ANCE, France.

Bertherat J. (1989), L'essaimage, levier de création d'entreprise, Rapport au Ministère du Travail, de l'Emploi et de la Formation professionnelle, Collection rapports officiels, $65 \mathrm{p}$.

Birley S. (1985), The Role of Networks in the Entreprenurial Process, Journal of Business Venturing, vol. 1, pp. 107-117.

Brenet P. (2000), Stratégie d'essaimage des grandes entreprises et création de PME, $5^{\text {ème }}$ congrès international francophone sur la PME, 25-27 octobre, Lille.

Bruyat C. (1987), Maîtriser l'essaimage peut être une condition importante de l'efficacité future de l'entreprise. Pour cela, il faut concilier les logiques de l'essaimeur et celles des essaimés, $8^{\text {ème }}$ journées nationales des IAE, Poitiers, 18-20 novembre, pp. 233-243.

Bruyat C. (1993), Création d'entreprise : contributions épistémologiques et modélisation, Thèse pour le doctorat ès Sciences de gestion, Université Pierre Mendés France, Grenoble II, 435 p.

Danvert L. (1990), L'essaimage ou la création d'entreprises par les salariés, Collection ANCE, Guide du professionnel, $196 \mathrm{p}$.

Daval H. (1998), L'essaimage : Analyse du processus entrepreneurial à travers le rôle de ses principaux acteurs, $7^{\text {ème }}$ conférence de l'AIMS, Louvain- la-Neuve, 27-29 mai.

Daval H. (1998), L'essaimage comme opportunité de création d'effets de synergie entre grandes entreprises et PME, Congrès international francophone sur la PME, Metz et Nancy, 22-24 octobre.

Daval H. (2001), Des processus d'essaimage différenciés: l'analyse des logiques des firmes essaimantes, $10^{\text {ème }}$ conférence de 1'AIMS, Québec, 13-15 juin.

Dennis W.-J. et Phillips B.-D. (1990), The Synergism of Independant High Tech Business Starts, Entrepreneurship and Regional Development, 2.

Everaere C. et Laviolette E.-M. (2006), L'essaimage en PME: une forme vertueuse de développement par excroissance, $23^{\text {ème }}$ Colloque annuel du conseil canadien des PME et de l'entrepreneuriat, Trois-Rivières.

Fayolle A. (2004), Entrepreneuriat : apprendre à entreprendre, Editions Dunod.

Husson G. (2004), Mission d'étude sur l'essaimage, Rapport pour le Ministre délégué aus petites et moyennes entreprises, au commerce, à l'artisanat et aux professions libérales et à la consommation, Avril.

Lafrance M. (1994), L'essaimage, un projet d'entrepreneurship entre les entreprises et leurs employés, Colloque sur la transformation des métaux légers, O.I.Q. régionale de la Mauricie \& Administrateurs agrées, Auberge Godefroy- Becancour, mai, 13 p.

Laviolette E.-M. (2003), Exploration des spécificités et des enjeux de l'essaimage en PME, $12^{\text {ème }}$ conférence de l'AIMS, Les Côtes de Carthage, 3-6 juin.

Laviolette E.-M. (2005), L'essaimage en PME: proposition d'un cadre d'analyse, Revue de l'Entrepreneuriat, vol.4, $\mathrm{n}^{\circ} 1, \mathrm{p} .53-68$. 
Laviolette E.-M. (2009), Enjeux et modalités de l'essaimage en PME, Humanisme et Entreprise, 2009/n²92, p.45-62.

Pirnay F. (1998), Spin-off et essaimage : De quoi s'agit-il ? Une revue de la littérature, $4{ }^{\text {ème }}$ colloque international francophone sur la PME, Metz et Nancy, 22-24 octobre.

Pirnay F. (2001), La valorisation économique des résultats de recherche universitaire par création d'activités nouvelles (spin-off universitaire) : Proposition d'un cadre procédural d'essaimage, Thèse de doctorat, Lille 2.

Sire B. (1988), L'essaimage : facteur d'émergence et de développement de la petite entreprise. L'exemple de la région Midi-Pyrénées, Cahier de recherche de l'IAE de Toulouse, 17 p. 